\title{
A numerical study of the first phase of a deep Mediterranean cyclone: Cyclogenesis in the lee of the Atlas Mountains
}

\author{
Kristian HORVATH*1, Lluis FitA ${ }^{2}$, ROMUALDO ROMERO ${ }^{2}$ and BRANKA IVANČAN-PICEK ${ }^{1}$ \\ ${ }^{1}$ Meteorological and Hydrological Service of Croatia, Zagreb, Croatia \\ ${ }^{2}$ University of the Balearic Islands, Palma de Mallorca, Spain
}

(Manuscript received August 1, 2005; in revised form December 14, 2005; accepted December 23, 2005)

\begin{abstract}
The initiation of a deep and severe impact Mediterranean cyclone in the lee of the Atlas Mountains is investigated by a series of numerical experiments using the MM5 forecast model. The roles of orography, surface sensible heat flux and upper-level potential vorticity anomaly are identified using the factor separation method. In addition, a sensitivity experiment addressing the role of a thermal anomaly in the lee of the Atlas is performed. The results of model simulations show that orography blocking is responsible for the generation of a low-level shallow vortex in the first phase of the lee development. An upper-level potential vorticity anomaly is the principal ingredient of this event, responsible for a dominant deepening effect in the later stage of lee formation. The analysis of the cyclone paths shows that orography tends to keep the cyclone stationary, while upper-level dynamical factors prove crucial for the advection of the system to the Mediterranean Sea. The most noteworthy influence of surface sensible heat flux is identified as an afternoon destruction of the surface baroclinic zone and the associated weaker cyclogenesis. Furthermore, it is shown that the thermal anomaly in the lee of the Atlas builds up rather quickly and tends to be responsible for the cyclone initiation positioning in the mountain lee.
\end{abstract}

\section{Zusammenfassung}

Die Anfangsphase einer großen Mittelmeerzyklone mit starkem Einfluss im Lee des Atlasgebirges wird in einer Reihe numerischer Experimente untersucht. Dabei wird das prognostische Modell MM5 angewendet. Die Rolle der Orographie, des Oberflächenflusses sensibler Wärme und der Anomalie der potenziellen vorticity wurde mithilfe der Factor Separations Methode ermittelt. Außerdem wurde ein Sensitivitätstest durchgeführt, um die Rolle einer thermischen Anomalie im Lee des Atlasgebirges festzustellen. Die Resultate der Modellsimulationen zeigen, dass die orographische Blockierung für das Entstehen eines flachen Bodenwirbels in der ersten Phase der Entwicklung einer Leezyklone verantwortlich ist. Eine Anomalie der potenziellen vorticity ist der Hauptbestandteil dieses Prozesses und verantwortlich für den dominanten Vertiefungseffekt in der späteren Phase der Leeentwicklung. Die Analyse der Trajektorie der Zyklone zeigt, dass die Orographie eine Tendenz dazu hat, die Zyklone stationär zu halten, während die dynamischen Höhenfaktoren für die Advektion des Systems zum Mittelmeer hin ausschlaggebend sind. Als wichtigster Einfluss des Oberflächenflusses sensibler Wärme wurde der nachmittägliche Abbau der baroklinen Bodenzone und die dazugehörige schwächere Zyklogenese identifiziert. Weiterhin wird gezeigt, dass sich die thermische Anomalie im Lee des Atlasgebirges ziemlich schnell entwickelt und für das anfängliche Positionieren der Zyklone im Lee des Gebirges verantwortlich ist.

\section{Introduction}

The purpose of this paper is to analyse the cyclogenesis in the lee of the Atlas Mountains, this being the first phase of a deep Mediterranean cyclone that caused a range of severe weather events throughout its movement over the Mediterranean Sea in November 2004.

North-West African (henceforth NWA) or Saharan cyclones are well known to influence the weather in the Mediterranean region. According to climatological studies approximately half of the cyclones that enter the Mediterranean region are initiated in North-West Africa

${ }^{*}$ Corresponding author: Kristian Horvath, Meteorological and Hydrological Service of Croatia, Gric 3, 10000 Zagreb, Croatia, e-mail: horvath@cirus.dhz.hr
(RADINOVIC, 1987). Although some of them originate in the southern Sahara, most are initiated in the lee of the Atlas Mountains (PEDGLEy, 1972). Despite the fact that a substantial number of NWA cyclones enter the Mediterranean Sea, from statistics done by CONTE (1985) it is clear that this region is far from a preferential one for deep cyclogenesis. Nevertheless, upon arrival above the Mediterranean Sea, a cyclone can be subjected a strong deepening and experience considerable growth-rates, reaching the ones associated with explosive cyclogenesis. In this way, possibly two of the most severe impact Mediterranean cyclones in last few years were initiated in the Atlas lee (Nov 2001, Nov 2004), this being one of the reasons for increased interest in NWA cyclogenesis. 


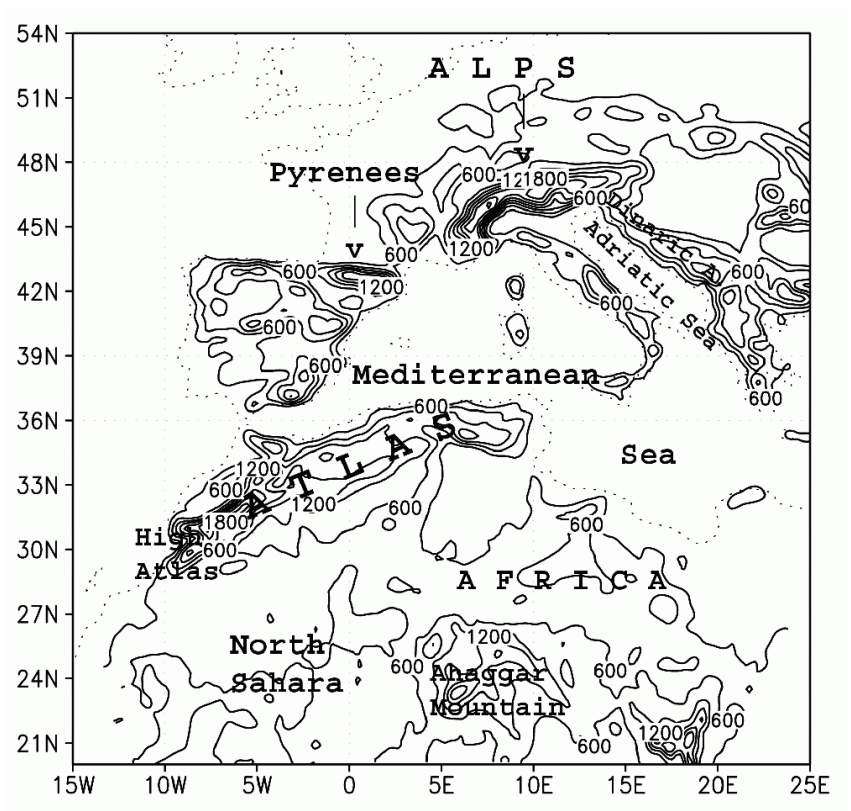

Figure 1: The Western Mediterranean and North-West Africa region with sites mentioned in the text. The area corresponds to a $24-\mathrm{km}$ resolution model orography in the domain, with contours every 300 meters, starting from 300 meters.

A strong intermonthly variability of NWA cyclone tracks was documented in ALPERT et al. (1990). According to the study, winter tracks are oriented northeast to north, characterised by cyclone protrusion to the Mediterranean Sea over the Tunisia area, while spring tracks tend to have a more pronounced eastern advection component. Probably the most extensive work on the description of synoptic conditions associated with NWA depressions was done by PREZERAKOS (1985, 1990) and PrEZERAKOS et al. (1990). In these studies, a detailed classification of different types of NWA depressions was established and their main characteristics discussed. The upper-level potential vorticity advection and a surface baroclinic zone were identified as the most important factors for the commencement of cyclogenesis for all cyclone categories (except the stationary ST category). The main mechanisms of deepening were proposed to be traditional Suttcliffe and Pettersen theories of development. Similar conclusions were derived in a work done by THORNCROFT and FLOCAS (1997). They propose that a scenario for a synoptic-scale NWA cyclogenesis is associated with the equatorward penetrating troughs at the end of the Atlantic storm track, followed by a 'finite amplitude' cyclogenesis.

On the other hand, a great number of studies analysing the Alpine lee cyclogenesis showed that Alpine lee cyclogenesis is a two-phase process (e.g. BUZZI and TiBALDI, 1978; MC GINLEY, 1982). The first phase is associated with a cold front retardation, a cold air outbreak into the Mediterranean Sea and a rapid creation of a shallow vortex in the Gulf of Genoa. The second phase fits the traditional baroclinic development and descriptive interpretation of a balanced low-levelupper-level vortex interaction. It follows from the invertibility principle (HoskINS et al, 1985) that any vortex contributing to the second phase should be either a thermal anomaly or a low-level PV anomaly. In recent studies (e.g. AEBISCHER and SCHÄR, 1998) it was argued that beside thermal anomaly, low-level PV anomaly created by flow deformation on the obstacle has a significant influence on the initiation and localisation of the lee cyclone. Namely, most of the Alpine lee cyclones tend to be bounded to the SW edge of the mountain, not being equally distributed in a mountain-sized thermal anomaly. However, influences of diabatic processes due to a proximity of this edge to the Mediterranean Sea and the known important role of moisture in Alpine lee cyclogenesis (e.g. Dell'OSSO and RAdinOviC, 1984; KUO et al., 1995) do not allow for a resolute conclusion. For this reason, many essentially 'non-precipitation' NWA lee cyclogenesis events could be a natural choice for testing the influence of parameters associated with dry lee dynamics. In this way, they could attribute with more confidence the dynamical roles of thermal and low-level $\mathrm{PV}$ anomalies in the process of lee cyclogenesis.

Thus, all of the aforementioned studies on the NWA lee (or Atlas lee) cyclogenesis basically relate to the second phase of lee cyclogenesis. The first-phase of NWA lee cyclones attracted little attention in a scientific literature, in both numerical and theoretical aspects. Up to our best knowledge, the only numerical study about this topic was done by EGGER et al. (1995). Simulating the cyclogenesis in the lee of the Atlas Mountains with idealised experiments where Atlas and Alps were introduced as polygons, they suggested that for an Atlas lee cyclone to form, it is sufficient that the orographic obstacle blocks the cold air and a strong PV anomaly moves over.

A Factor Separation (FS) method aiming to evaluate the influences of different factors as well as their synergies in the analysed process proposed by STEIN and ALPERT (1993) was widely applied to case studies (e.g. AlPERT et al., 1995; AlPERT et al., 1996; GUAN and REUTER, 1996; KRICHAK et al., 1997a, b; ROMERO et al, 1997; BERGER, 2001; ROMERo, 2001). AlPERT et al. (1996) investigated the multi-stage evolution of an Alpine lee cyclone testing the roles of topography, convection and sensible and latent heat fluxes. Their results quantitatively showed the cyclogenetic dominance of topography blocking, convection, convection induced by orography and surface fluxes and local moisture flux in four subsequent time periods. More recently, TSIDULKO and ALPERT (2001) addressed the roles of upper-level potential vorticity and orography (as well as their synergy) on the case of Alpine lee cyclogenesis. They showed that pure upper-level potential vorticity in- 

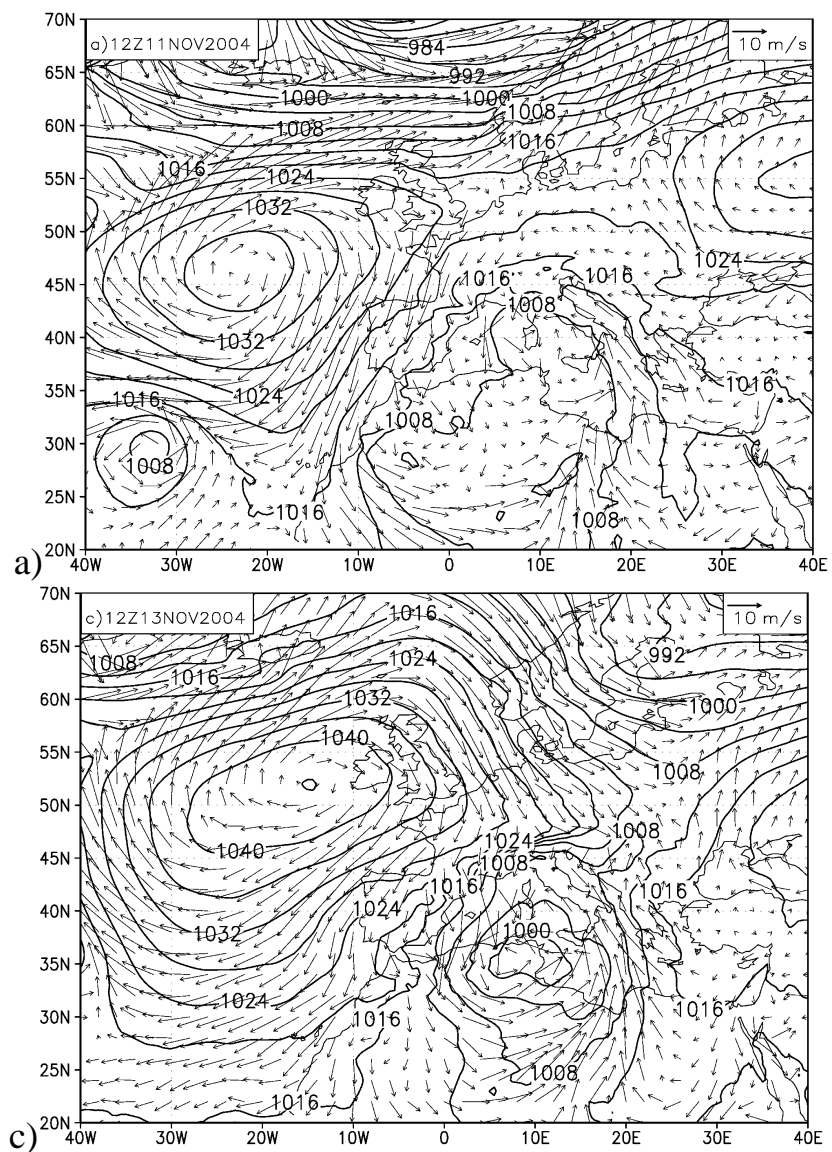
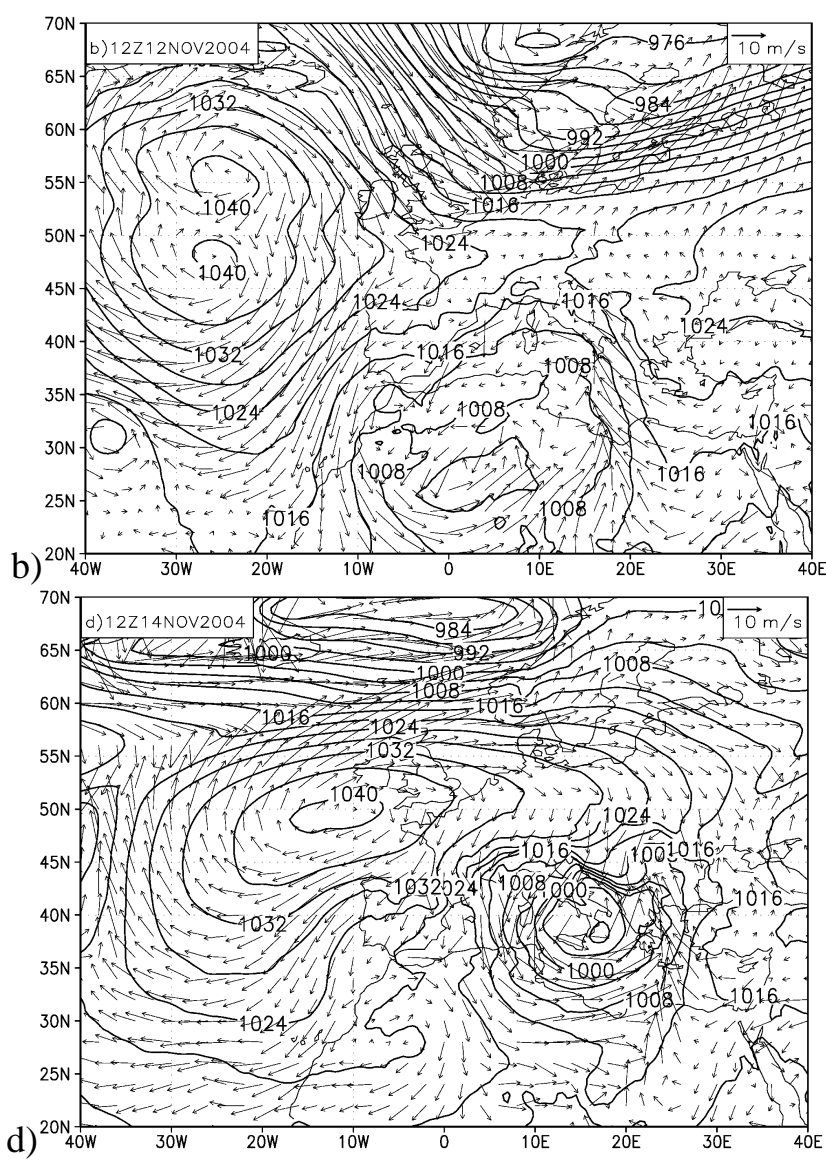

Figure 2: Surface synoptic situation showing mean sea level pressure and $10 \mathrm{~m}$ wind vectors over Europe and the Eastern Atlantic at 11 Nov 200412 UTC (a), 12 Nov 200412 UTC (b), 13 Nov 200412 UTC (c) and 14 Nov 200412 UTC (d).

fluence plays the most significant role in the cyclone initiation and deepening.

In this study, for the first time the FS method will be applied to a case of Atlas lee cyclogenesis (Fig. 1), investigating the influence of orography, sensible heat flux and upper-level potential vorticity anomaly as well as their mutual interactions. In this way, contributions of selected factors on cyclone deepening will be quantified and an attempt to test numerically the two-phase development of lee cyclones in the Atlas area will be made. It will be shown that these factors not only modified the cyclone intensity, but also significantly altered the cyclone track. As a result of the analysis, one of the potential contributors to the seasonal cyclone track variability will be identified. Finally, the sensitivity study will address the role of a thermal anomaly in a separate sensitivity experiment, as an attempt to separate the influences of a thermal anomaly and a low-level, orographically induced potential vorticity, on the low-level vortex generation.

The paper is organised as follows: Section 2 gives the observational evidence of the analysed case. A model description and a brief overview of the factor separation method and the simulations performed will be given in Section 3 . The control run will be described and verified in Section 4, followed by the presentation of factor separation results and the related discussion. Section 5 is devoted to the sensitivity study on the role of a thermal anomaly, while concluding remarks are summarised in Section 6.

\section{Synoptic environment}

The observational data in the Sahara region is rather sparse, so usually reliance is made on the ability of the model to forecast the regional weather events. However, for the purpose of analysis and model verification, we will use the surface analysis of the European Centre for Medium Range Weather Forecasts to investigate the low-level evolution of the cyclone. On the other hand, at upper levels satellite pictures will be used to address the relevant upper tropospheric circulation features.

The synoptic setting, within which the range of severe weather events over the Mediterranean area developed from 13 to 15 November 2004, followed a deep cyclogenesis initiated in the lee of the Atlas Mountains. The synoptic pattern at low levels was dominated by a stationary Azore anticyclone producing strong northerly winds over the eastern Atlantic and Portugal area. At the same time, a moderate, zonally oriented quasi-stationary 


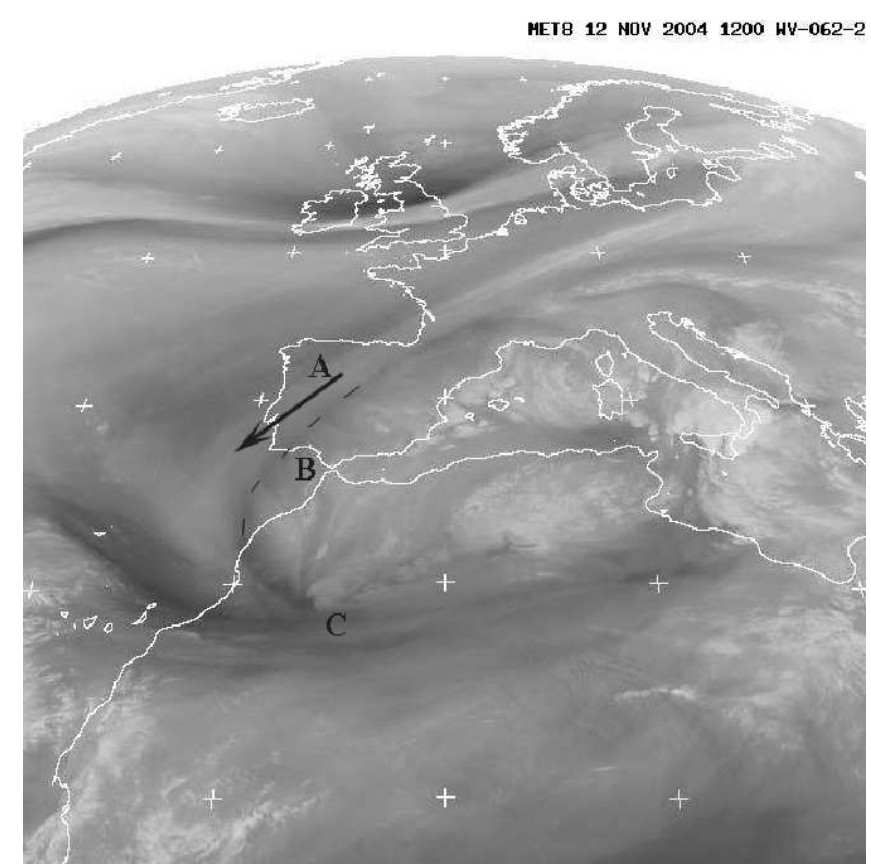

Figure 3: Water-vapour Meteosat MSG imagery for 12 Nov 2004 12 UTC showing an upper level trough over Western Europe and North Africa. The arrow (A) denotes the jet stream, the dashed line (B) the associated dark stripe, while the point $\mathrm{C}$ denotes the cyclone initiation point.

baroclinic zone over the North-African coast started to deform, creating an Atlas-sized thermal anomaly. After a short retardation, cold air crossed the lower NE part of the Atlas range and penetrated southward (Fig. 2.a). In subsequent hours, the Azore high and associated winds near the SW Atlas edge intensified. Along with a high surface heat flux impact in the arid mountain lee (a model simulation value reaching $400 \mathrm{Wm}^{-2}$ ), this situation led to creation of a more localised warm thermal anomaly and associated depression in the lee of the High Atlas Mountains, also indicated by an orographic pressure perturbation distribution above the area (Fig. 2.b). Near the SW mountain edge, a strong shear line extended deep into the lee, indicating the region of an enhanced low-level PV anomaly and an increased surface baroclinicity. In the subsequent hours, a shallow lowlevel vortex gradually started to build up moving to the north-east. On 13 Nov 12 UTC (Fig. 2.c), the cyclone centre already approached the Mediterranean, where it was quickly advected eastward, continued to deepen and caused a range of severe weather events. This lowpressure centre, combined with the anticyclone, created a strong north-south pressure gradient over the Alpine and Dinaric Alpine regions (Fig. 2.d). These synoptic conditions, generally conducive for the development of Mistral and flow deflection around the eastern edge of the Alps leading to the onset of Bora wind, gave rise to severe mesoscale Bora phenomenon along the Eastern Adriatic coast. Measurements showed the existence of sustained winds of $35 \mathrm{~ms}^{-1}$ over the 24 hour interval over a wide area of the Eastern Adriatic coast and gusts occasionally reaching $60 \mathrm{~ms}^{-1}$, what were one of the highest Bora gust values ever recorded.

The synoptic situation at upper levels is shown on the EUMETSAT water vapor imagery (Fig. 3), which is strongly linked to the upper-level potential vorticity distribution (e.g. LAGOUVARDOS and KOTRONI, 2000; MANSFIELD, 2001). A strong upper-level trough extended over the wide Mediterranean and Western European region. A dark stripe on the figure indicates the subsidence area on the cyclonic part of the jet stream, identifying the area of a very low water vapor content and the highest upper-level PV values. Near the southern end of the dark stripe, above the lee of the High Atlas, evidence of the early stage of cyclogenesis can be noticed. As inspected from the satellite picture series, after southward advection to the area above the Atlas Mountains, the trough was advected north-eastward towards the Mediterranean region. On $14 \mathrm{Nov}$, in the maturing stage of the cyclone development, a strong frontal activity can be identified over southern Italy on the highresolution visible spectra satellite image (not shown), indicating the existence of heavy flood-raising precipitation that reached $200 \mathrm{~mm} / 24 \mathrm{~h}$.

\section{Description of experiments}

\subsection{Model description}

The simulations were performed with a non-hydrostatic version of the fifth generation Pennsylvania State University - National Center for Atmospheric Research mesoscale model MM5 (DUDHIA 1993, GRELL et al. 1994). The model uses a terrain following vertical coordinate with enhanced vertical resolution in the boundary layer to better represent the turbulent processes in atmospheric boundary layer. The horizontal grid has an Arakawa-Lamb B-staggering of the velocity variables with respect to the scalars. For this study a domain of $160 \times 160$ points was chosen centred at $5.0 \mathrm{E}$ and $37.0 \mathrm{~N}$, with 23 vertical levels and $24 \mathrm{~km}$ resolution. Simulations were initialised on 11 Nov 200400 UTC and run for the 72 hours period.

Initial and boundary conditions were provided by the global NCEP/NCAR (National Centers for Environmental Prediction/National Center for Atmospheric Research) Final Analysis (FNL). They were horizontally interpolated to the model resolution and enhanced by assimilation of surface and upper-air NCEP observational data sets. The planetary boundary layer parameterisation used a modified version of the BLACKADAR (1979) scheme (ZHANG and ANTHES 1982; ZHANG and FRITSCH, 1986) parameterisation. Other physical parameterisations included the BETTS and MILlER 

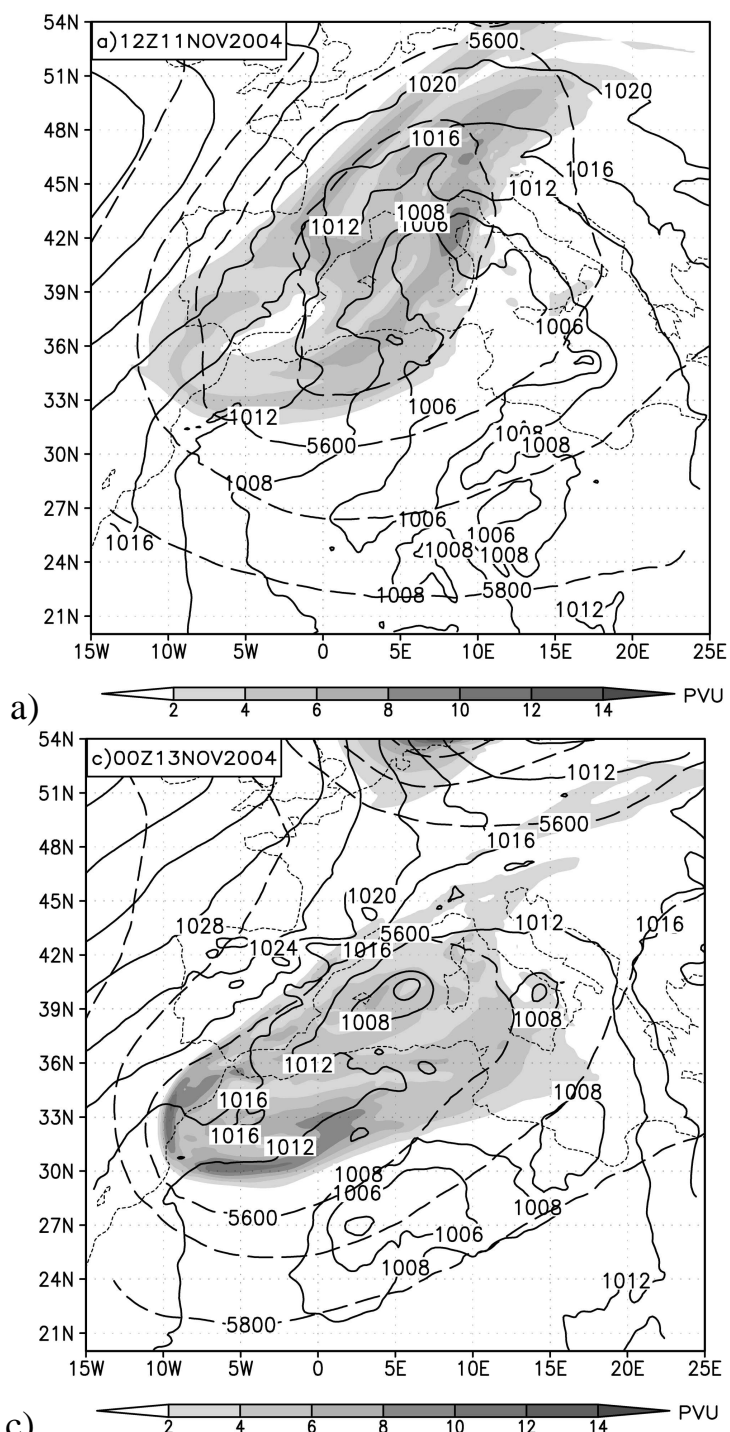

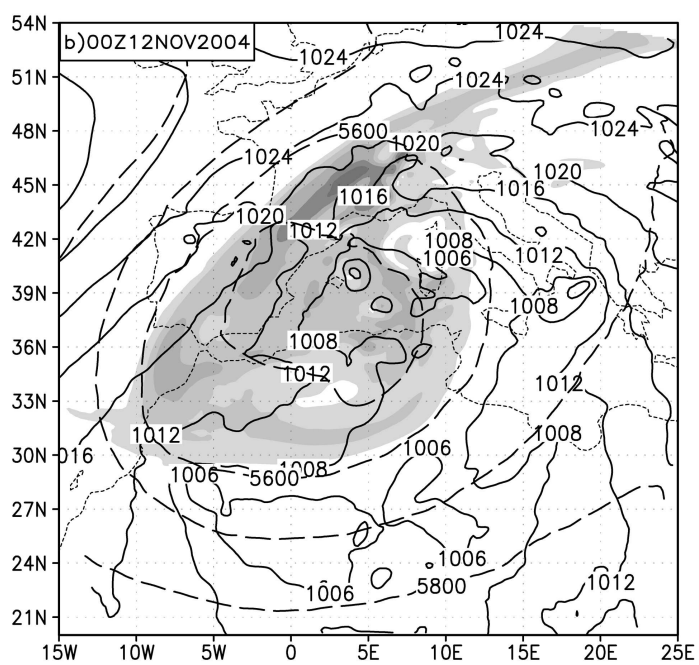

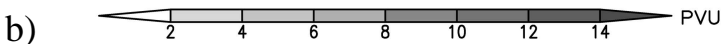

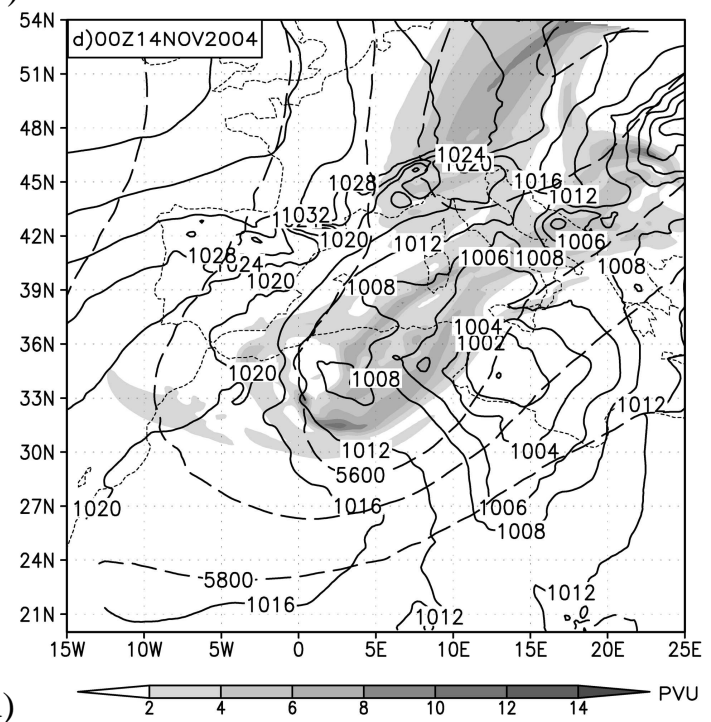

Figure 4: Model forecast of synoptic scale evolution at 11 Nov 200412 UTC (a), 12 Nov 200400 UTC (b), 13 Nov 200400 UTC (c) and 14 Nov 200400 UTC (d). Mean sea level pressure contours are plotted in continuous line with $4 \mathrm{hPa}$ above $1008 \mathrm{hPa}$ and $2 \mathrm{hPa}$ below $1008 \mathrm{hPa}$ values. Upper-level potential vorticity values at $300 \mathrm{hPa}$ are shaded (white under $2 \mathrm{PVU}$ black over 14 PVU), while geopotential at $500 \mathrm{hPa}$ is plotted in dashed (every $100 \mathrm{gpm}$ ).

(1993) cumulus parameterisation, simple-ice explicit moisture scheme and cloud-radiation scheme, while for soil parameterisation the multi-layer soil temperature model (DUDHIA, 1996).

\subsection{Method description}

The Factor Separation (FS) method allows us to quantify not only the contributions of the sole factors, but also their mutual synergies. It is a useful approach if interactions are expected among the investigated factors. To evaluate the influence of $n$ factors and their mutual synergies it is necessary to run $2^{n}$ model simulations. In order to illustrate the method fully described in STEIN and ALPERT (1993), a single pure factor, double synergy and triple synergy effects are listed below for reference:

$$
F_{i}=f_{i}-f_{0}
$$

$$
F_{i j}=f_{i j}-\left(f_{i}+f_{j}\right)+f_{0}
$$

$$
F_{i j k}=f_{i j k}-\left(f_{i j}+f_{i k}+f_{j k}\right)+\left(f_{i}+f_{j}+f_{k}\right)-f_{0}
$$

where $f_{i j k}\left(f_{i j}\right.$ or $\left.f_{i}\right)$ denotes a value of the predicted field in simulation when factors $i, j$ and $k(i, j$ or $i$ respectively) are switched on. $F_{i}$ is a part of a predicted field due to a sole contribution of a factor $i$ and $F_{i j k}\left(F_{i j}\right)$ is a part of a predicted field due to an interaction of the factors $i, j$ and $k(i, j)$ only. $f_{0}$ denotes a so-called background simulation when all analysed factors were withheld (switched off) from the simulation.

The factors chosen in this study are the Atlas orography, an upper-level PV anomaly and surface sensible heat flux. Whilst the first two factors clearly influence 

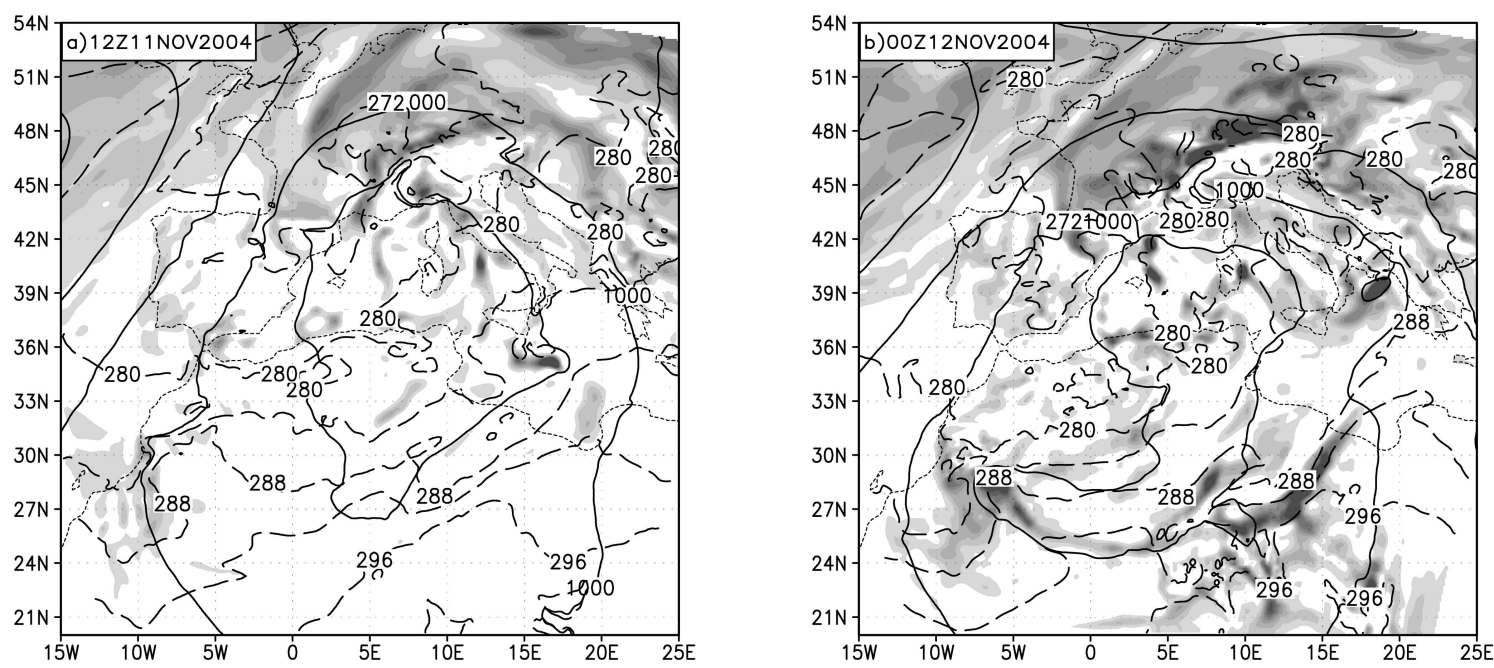

a)

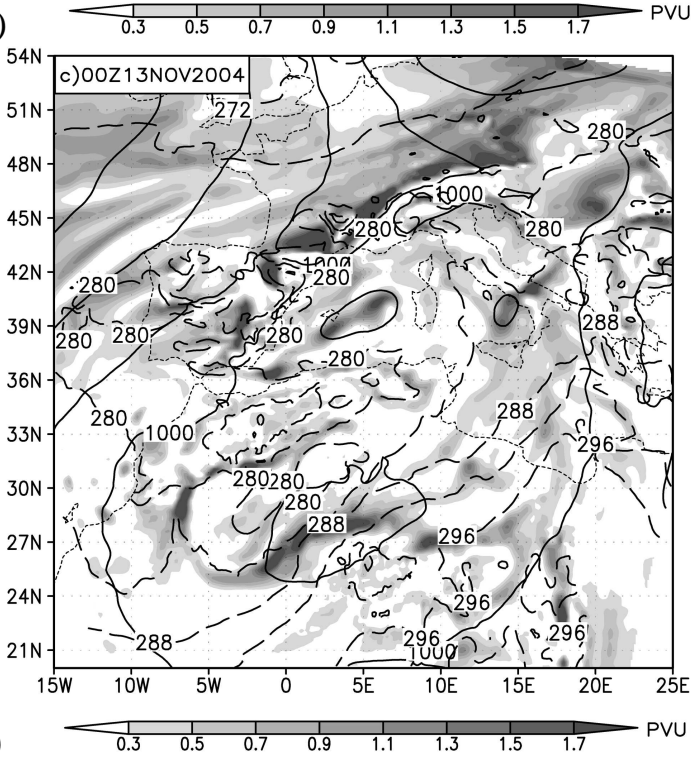

b)

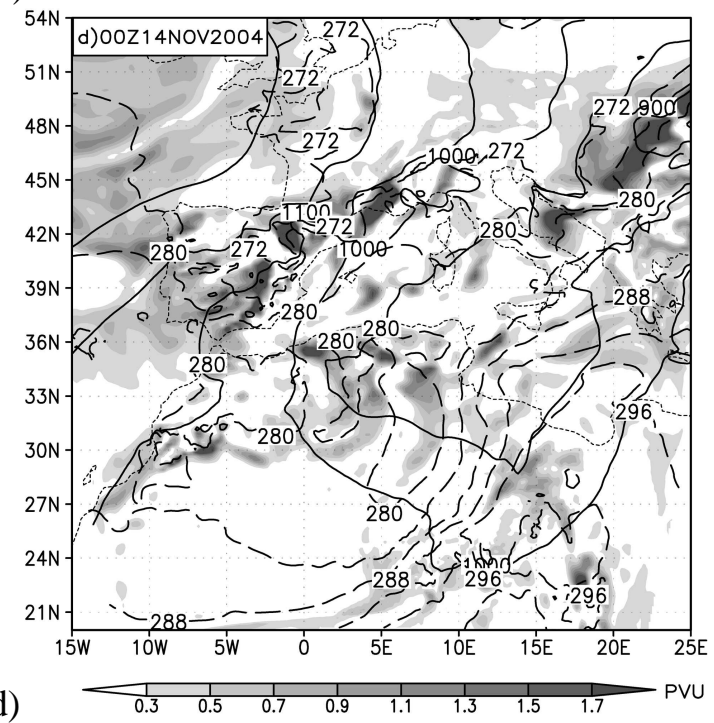

Figure 5: Model forecast of low-level conditions at $925 \mathrm{hPa}$ at 11 Nov 200412 UTC (a), 12 Nov 200400 UTC (b), 13 Nov 200400 UTC (c) and 14 Nov 200400 UTC (d). Low-level potential vorticity is shaded (white under 0.3 PVU and black over 1.7 PVU). Geopotential is in continuous line (every $50 \mathrm{gpm}$ ), while temperature is in dashed (every $4 \mathrm{~K}$ ).

the cyclone formation and deepening in line with general theory of lee cyclogenesis, surface sensible heat flux was chosen to shed light on its specific influence in the arid Saharan region of the Atlas Mountain lee.

\section{Numerical simulations}

\subsection{Control simulations}

The synoptic evolution of the lee cyclone in the control simulation (Fig. 4.a-d) shows that mean sea level pressure fields, system movement and deepening were moderately well simulated, experiencing some time delay in the process evolution. The upper-level potential vorticity core at $300 \mathrm{hPa}$ level reached $14 \mathrm{PVU}$ at the time it was advected over the Atlas region (Fig. 4.c). This cutoff upper tropospheric low induced a closed upper-air circulation as indicated by geopotential height distribution. Towards the end of the analysed period (Fig 4.d), which is approximately the time when the cyclone left the lee and entered the Mediterranean (14 Nov 00 UTC), the upper-level potential vorticity anomaly weakened, probably at the expense of interaction with the low-level vortex. The axis of the lee cyclone had a slightly negative tilt that tended to get more neutral with time. All of the aforementioned facts agree with the PETTERSEN and SMEB YE (1979) cyclone type B, and are in close resemblance with the lee cyclogenesis synoptic features.

In a mesoscale analysis of the model run, we investigated the lower tropospheric conditions. As mentioned in Section 2, strong northerly winds together with high surface sensible heat flux created an Atlas-sized thermal anomaly already on 10 Nov, far prior to the cyclogenesis. However, soon upon its creation, cold air crossed the lower NE part of the Atlas range and reduced the thermal anomaly to the lee of the High Atlas Mountains (Fig. 5.a). A strong shear line is visible on 12 Nov 00 


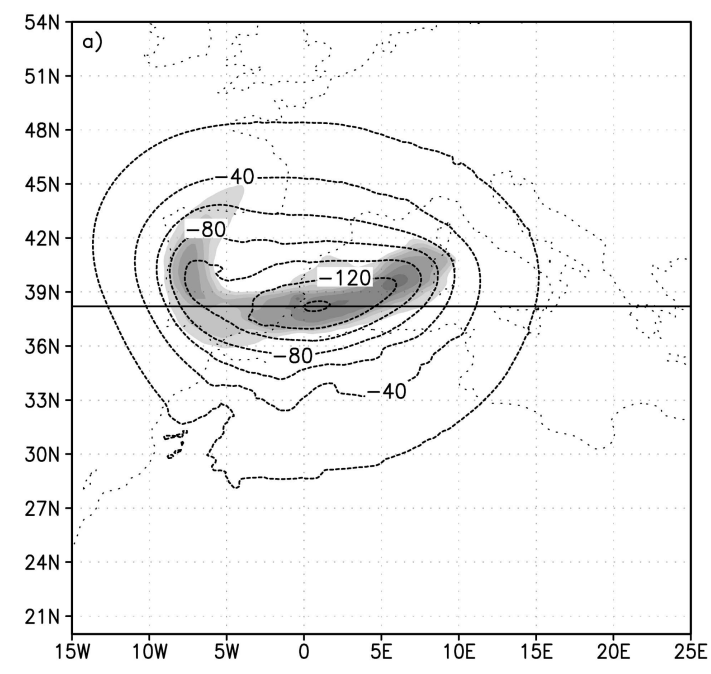

a)

Figure 6: Definition of the upper-level potential vorticity perturbation at $300 \mathrm{hPa}$ subtracted from the initial conditions at 11 Nov 200400 UTC, in shaded (a). The solid line denotes the position of the vertical cross-section through the perturbation (b). The associated geopotential perturbations at the $300 \mathrm{hPa}$ level and in the vertical cross-section are superimposed in dashed line.

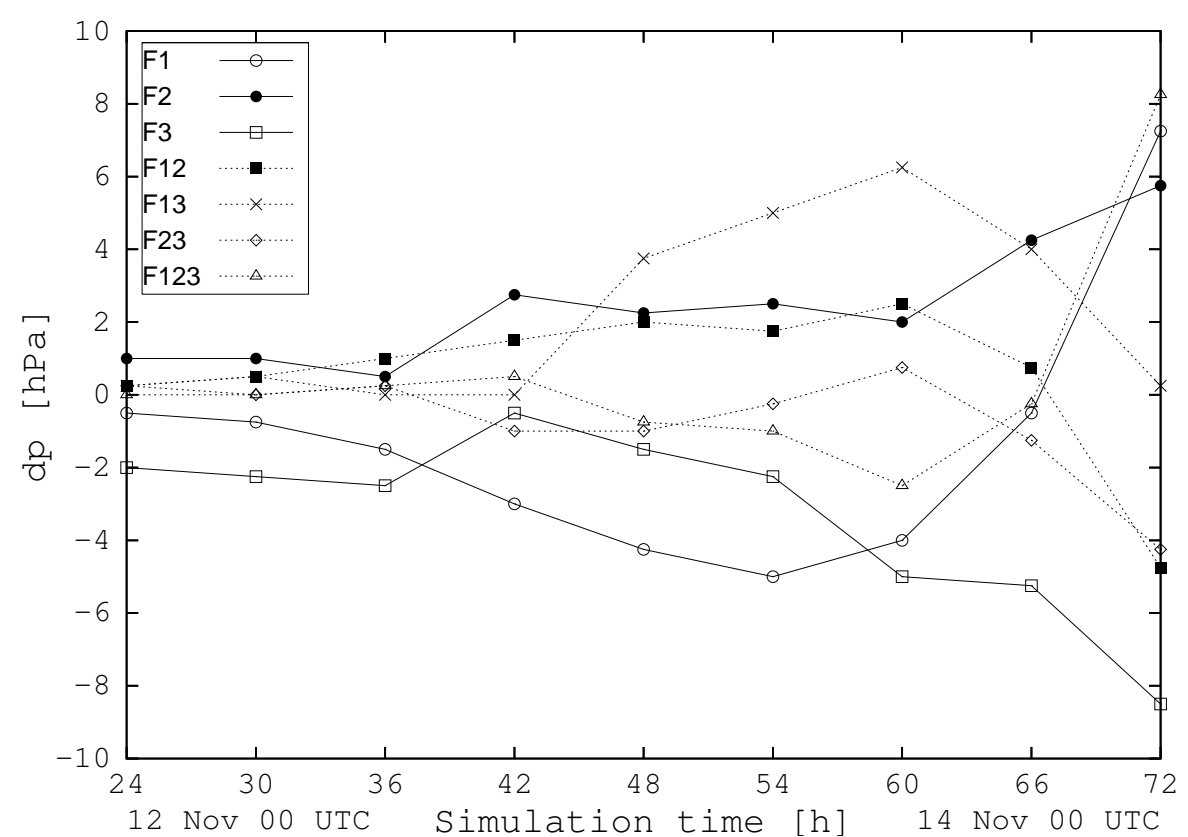

Figure 7: The 48-hour time evolution for 7 local contributions to the cyclone deepening (hPa), after 24 hours of simulation. F1, F2 and F3 are influences of orography, surface sensible heat flux and the upper-level PV perturbation (refer to Fig. 6), respectively, plotted in continuous lines. Contributions of synergies are plotted in dashed lines (e.g. F13 denotes the contribution of a synergy between orography and upper-level potential vorticity perturbation).

UTC as primary orographic PV banner of a considerable horizontal distance starting over the SW Atlas edge and overcoming 2.5 PVU in intensity (Fig. 5.b). At the same time, two frontal low-level PV sources are visible: the first one corresponding to the front that crossed the central and the northern part of Atlas and the second one more away to the east. They belonged to the same initial baroclinic zone and were separated due to the temporary orographic blocking of the first and quick outbreak from the Mediterranean of the second. In this period and sub- sequent hours, the very cyclone centre was located in a small quasi-barotropic zone and an area of enhanced low-level PV. During the $12^{\text {th }}$ of November, the cyclone moved northward towards the Mediterranean area. Lowlevel PV banners weakened, as a mere consequence of the reduced daytime stability. At 13 Nov 00 UTC (Fig. 5.c), the orographic primary PV banner merged with the frontal one, forming a continuous source of low-level PV far away from the mountain. At the time, the cyclone centre started to be attached to the frontal baroclinic 
zone and stayed that way until the end of the analysed period. Orographically induced PV banner seemed to feed the low-level cyclone, resembling some of the documented cases of Alpine lee cyclogenesis (AEBISCHER and SCH ÄR, 1998). On 14 Nov 00 UTC (Fig. 5.d), which is the last time-sequence of our analysis, roughly corresponding to the time the cyclone left the lee and entered the maritime area, the cyclone centre reached $1000 \mathrm{hPa}$ depth and was characterised with a well defined frontal structure.

Despite of the fact that differences between model simulations and ECMWF analysis reveal that the model simulation of the investigated process was of limited quality, it is believed that its features were captured reasonably well enough to make the case appropriate for the sensitivity study and deduction of factor contributions in this lee cyclogenesis event.

\subsection{Factor separation results and discussion}

In previous sections upper-level potential vorticity (PV), orography and surface sensible heat flux (SSHF) were identified as main factors affecting this cyclogenesis event. In this section we will address qualitatively the contributions of these factors and their mutual synergies to the mean sea level value and cyclone centre position. As mentioned in section 3.2, for that purpose 8 simulations had to be done. Thus, to address the orographic contributions to the process, the Atlas Mountains were removed from the terrain field. Secondly, surface sensible heat flux was withheld in a series of simulations, allowing for a determination of the influence of the associated surface forcing. Finally, an upper-level potential vorticity anomaly was removed from the initial conditions to address the role of upper-level dynamical processes in the cyclogenesis.

The removal of the upper-level potential vorticity anomaly from initial conditions was performed by applying the piecewise PV inversion scheme (DAVIS and EMANUEL, 1991) on Ertel's potential vorticity fields. The balance condition introduced in the scheme was the non-linear balance condition (CHARNEY, 1955). The inversion was performed at the simulation starting time (11 Nov 00 UTC) using the NCEP/NCAR isobaric analysis on the model resolution and domain. The PV anomaly was defined as the departure from the 10-day time average (centred at 12 Nov 00 UTC) PV field between $500 \mathrm{hPa}$ and $100 \mathrm{hPa}$ levels. However, in simulations with a total upper-level PV anomaly removed, the cyclone was excessively changed, both in intensity and in path. Thus, in order to keep the similarity with the real case, a PV perturbation used for our sensitivity study addressed only half of the total PV anomaly (Fig. 6.a). This later resulted in an upper-level PV anomaly that e.g. on 12 Nov 06 UTC reached 8 PVU at 300 $\mathrm{hPa}$ level, compared to the almost $14 \mathrm{PVU}$ associated

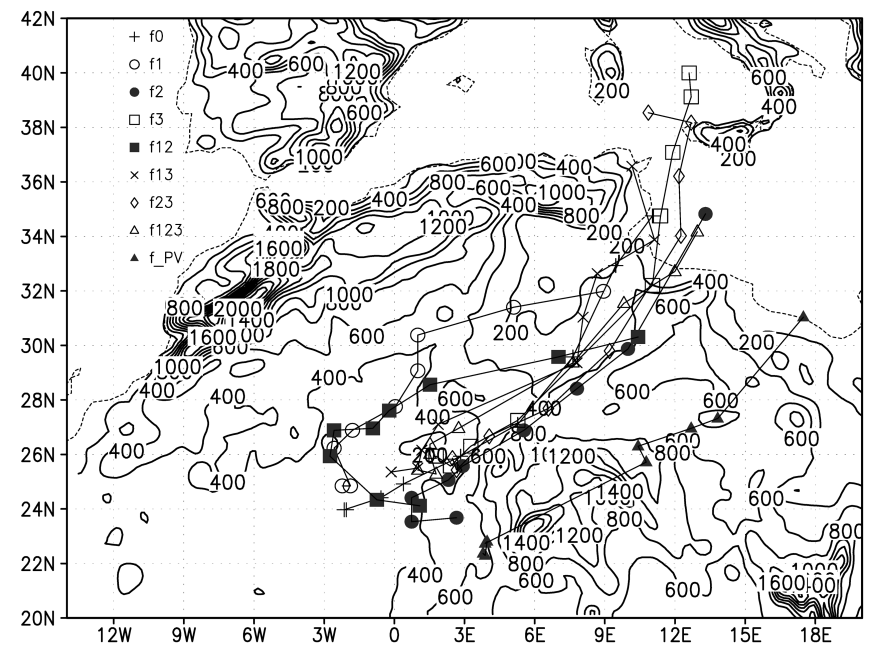

Figure 8: Time evolution of the cyclone centre in nine simulations, starting from 12 Nov 200400 UTC, plotted every 6 hours. f0 is the simulation with all three investigated factors withheld, and f_PV is the simulation with the total upper-level potential vorticity anomaly subtracted from the initial conditions (refer to text). Other simulation denotations are described in text (chapter 3.2).

with the original upper-level PV anomaly. This should be kept in mind when considering the quantitative contributions of the upper-level potential vorticity and its interactions. Moreover, it should be noted that the upperlevel PV perturbation removal influenced the state of the whole troposphere (Fig. 6.b).

A time evolution of the factor contributions to the mean sea level pressure value illustrates the dominance of different processes at different stages of the cyclone development (Fig. 7). The analysis focused on the period starting from 12 Nov 00 UTC (24 hours after the simulation initiation) that is the approximate time of cyclogenesis commencement in the simulation. The apparent cyclogenetic influence of upper-level PV in the start of the analysed period (12 Nov 00 UTC) probably merely reflects the change in initial conditions. Namely, a removal of upper-level PV perturbation caused a warming of the low-level cold air impinging on the Atlas, thus reducing the intensity of the thermal anomaly and the associated pressure low. That is the reason why, before the cyclogenesis commenced, a mean sea level pressure was somewhat higher in the background simulation (with all factors excluded) then in the simulation with the upperlevel PV perturbation included.

The first pronounced cyclogenetic influence was an orographic one, starting around 12 Nov 00 UTC, cumulatively exerting $5 \mathrm{hPa}$ deepening on the cyclone. This influence was associated with a frontal retardation and creation of the thermal and low-level PV anomalies in the lee of the High Atlas that were not present in simulations without orography. At that time and in subsequent hours, the cyclone centre was mostly attached to the quasi-barotropic lee area, marking the first phase of 
the lee cyclogenesis. At 13 Nov 06 UTC, an orographic influence first diminished, and then became strongly cyclolytic (destruction of a cyclone). This type of duality of orographic influence was already noticed in a study of the Alpine cyclogenesis by ALPERT et al. (1996) and was probably due to cyclone movement out of the favourable lee area. Similar results were achieved in TSIDULKO and ALPERT (2001), although the cyclogenetic influence of orography in their analysis does not seem to be so intensive as in our study.

The upper-level potential vorticity perturbation started to contribute to cyclogenesis at the time its strongest core got advected over the Atlas lee at 12 Nov 18 UTC. Its influence was in the subsequent period associated with reduced stability of the troposphere and a creation of a slightly stronger thermal anomaly at the surface (inclusion of the upper-level PV perturbation cooled the air impinging on the Atlas). Furthermore, stronger positive vorticity advection at upper levels induced greater low-level vertical velocities (e.g. in accordance with the quasi-geostrophic omega equation) and stronger low-level convergence, resulting in a more intense low-level vortex development. Stronger winds at lower levels resulted in the more intense cyclonic circulation and the stronger shear line in the lee. In other words, a cyclonic circulation of the upperlevel potential vorticity anomaly propagated vertically (downward) to the middle and lower atmosphere, in accordance with conclusions derived from similar experiments for the Alpine lee cyclogenesis (TSIDULKO and ALPERT, 2001) and in idealized numerical experiments on the Sharav cyclone (EGGER et al., 1995) However, as later analysis is going to show, the difference between the speeds of advection in simulations that define the upper-level PV perturbation influence (refer to Eq. 3.1) indicates that part of its strong cyclogenetic influence in the end of the analysed period could be partially related to moist processes over the Mediterranean Sea.

The most pronounced contribution of sensible surface heat flux seems to be the afternoon cyclolysis. It is evident that surface heat flux enables heat exchange between the atmospheric boundary layer (ABL) and the surface. Thus, in the simulation without surface sensible heat flux, absorbed energy during the daytime (when SSHF is directed upwards) caused higher ground temperatures, but lower surface air temperatures near the ground and a less intensive atmospheric boundary layer. In this environment, a weaker ABL and less vertical mixing contributed to a stronger baroclinic zone and more intensive cyclone deepening. A similar cyclolytic influence in the early stage of the cyclone development has been noticed in work done by KUO et al. (1991), although their case took place over the sea. Furthermore, a horizontal air temperature gradient in the surface baroclinic zone was stronger then the horizontal ground tem- perature gradient at the same location. Thus, when enabled, the heat exchange tended to increase the horizontal temperature gradient in the ground, and decrease it in the low-level atmosphere, weakening the baroclinic zone and cyclone deepening. Finally, it is proposed that exclusion of SSHF affected the radiation balance at different levels in the atmosphere. In absence of SSHF, during the daytime, ground temperature was higher then in the presence of SSHF. This situation enhanced the longwave earth radiation, which became the main heat exchange factor between the ground and the atmosphere, since latent heat flux over the arid Atlas lee was rather small. In contrast to SSHF that transferred the heat more locally to the surface boundary layer (and indirectly to the whole ABL through turbulent mixing), long wave terrestrial radiation was absorbed the most where the humidity was the highest. Throughout this process, cloudy areas in the free troposphere gained more, while the surface layer gained less heat energy compared to the control run. Thus, the ABL was less intensive contributing to a stronger surface baroclinicity and cyclogenesis as described above. On the other hand, the moist free troposphere was less stable due to increased heating. The heating of the moist free troposphere is known for its strong contributing to a cyclone deepening. For example, in ALPERT et al. (1996), latent heat release was identified as one of the main contributors to cyclone deepening. Although the underlying mechanism was clearly not the same, the final effect in terms of a heat supply to the free troposphere seems to be qualitatively similar.

The afternoon cyclogenetic effect of SSHF noticed in our study seems to oppose the findings of ALPERT and ZIV (1989), who investigated the generation mechanism of the Sharav cyclones with modified two-level linear baroclinic model suggested by PHILLIPS (1954). Thus, their study mostly considered global to regional scale analysis that can be captured by quasi-geostrophic balanced dynamics. This result seems to indicate the scale dependence of the SSHF influence, and the importance of mesoscale frontal dynamics and boundary layer processes representation in the model, in order to fully determine the role of surface sensible heat fluxes in lee cyclogenesis events.

It is interesting to note the contribution of the synergy between orography and upper-level potential vorticity on the mean sea level pressure values. The influence was clearly cyclolytic during the 18 -hour period, starting from 12 Nov 18 UTC. It will be shown later in the analysis of cyclone paths, that it seems to be connected to the cyclone destruction at the orographic obstacle on the way to the Tunisia area. After that, on 13 Nov 12 UTC and subsequent hours, the synergy contributed to the cyclone recovery. At that time lower and upper-level vortices were tilted to the favourable western 

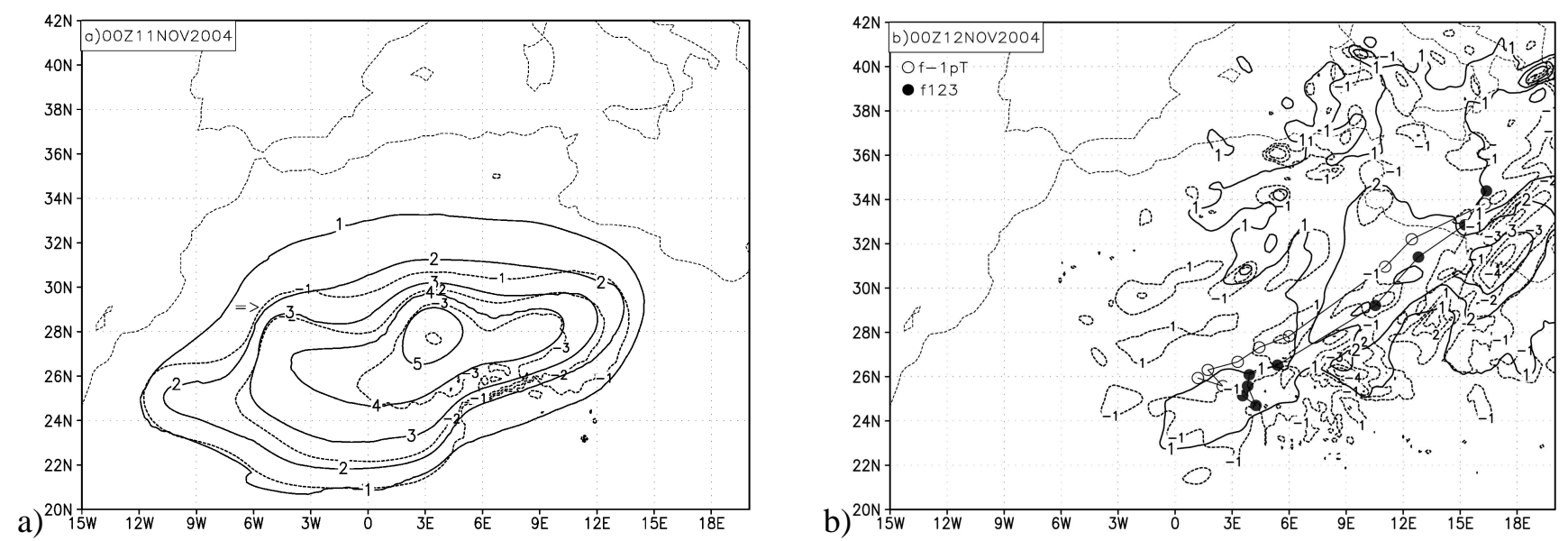

Figure 9: Definition of the thermal anomaly subtracted from the initial conditions at 11 Nov 200400 UTC (a). Fields denote a difference between the modified initial fields for the thermal anomaly sensitivity experiment and the original initial fields of the control run. The initially positive mean sea level pressure modification $(\mathrm{hPa})$ is plotted in continuous line and the initially negative temperature modification at $925 \mathrm{hPa}$ in dashed. The arrow denotes the lee of the High Atlas mountains and removal of the 'secondary' thermal anomaly. The structure of the thermal anomaly perturbation after 24 hours of simulation at 12 Nov 200400 UTC (b), defined as a difference described above. The cyclone trajectories in the experiment with thermal anomaly removed (f-1pT) and in the control run (f123) were superimposed on the figure, starting from 12 Nov 00 UTC, plotted every 6 hours.

direction with height. Rough estimates of geopotential at $300 \mathrm{hPa}$ and mean sea level fields on 13 Nov 12 UTC gave a horizontal dimension of the upper-level trough wave of $\mathrm{L}=60^{\circ}$, and a separation of the centres of around $0.15 \mathrm{~L}$. If these numbers were applied to the idealised conceptual models of low-level-upper-level vortex interaction or linear instability theory (BRETHERTON, 1966; Hoskins et al., 1985), the waves would tend to hold themselves against the zonal flow (i.e. against differential advection), growing and reinforcing each other. This qualitative consideration seems to be roughly applicable at the end of the analysed period, when low-level and upper-level centres tended to be almost locked in phase.

It has been observed that an inclusion of SSHF in the simulations strengthens the upper-level PV. This is in qualitative agreement with the cyclogenetic synergy that SSHF and upper-level PV seemed to show in the afternoons. Other synergies did not seem to exert a significant impact on the cyclone centre deepening, except in the very end of the analysed period. The corresponding results at that time should be interpreted with care, because of the cyclone centre spread at the end of the simulations. Namely, in some simulations the cyclone centres entered the Mediterranean, while in others still stayed on the continent. In this way, possible moisture processes could have influenced the results. However, the role of moist processes is out of the scope of this paper and will not be analysed here.

Cyclone centre paths in factor separation simulations are shown on Figure 8. A cyclone centre path for the simulation with the total upper-level PV anomaly removed from the initial conditions is added to the picture. Considering the latter first, we can see that without upper-level PV anomaly in the initial conditions the cyclone initiation point and movement were excessively changed. The shallow cyclone was indeed formed in the lee of the Ahaggar Mountain (refer to Fig. 1), dominated by a weak advection of the upper-level PV from the boundary conditions over that area. Thus, the analysis suggests that strong upper-level PV advection is crucial for the cyclogenesis in the lee of the Atlas to occur.

The influence of orography on cyclone paths was clearly resolved - the four closest cyclone paths to the Atlas range corresponded to the four simulations with orography included. Therefore, in the first place orography tended to move the location of cyclone initiation to the favourable lee area where orographically induced low-level PV and thermal anomalies were the strongest. In addition, it continued to keep the cyclone path deeper inside the lee area. In contrast, inclusion of the upperlevel PV perturbation moved the position of the cyclone formation away from the mountain. It seems that the stability of the lower atmosphere on the windward side of the Atlas played an important role in localising the formation place. Namely, in simulations with the upperlevel PV perturbation included, the lower atmosphere was less stable underneath. A closer look reveals that in those simulations cold air parcels on the windward side were able to cross the mountain more efficiently and moved the thermal anomaly more to the south-east. Adversely, in simulations without the upper-level PV perturbation, the low-level stability was stronger and the air was forced to go around the obstacle, creating a thermal anomaly deeper inside the mountain lee. Diffluence of the cyclone paths in simulations with a variable intensity of the upper-level PV anomaly indicates that upper- 
level dynamical factors have a potential to account for the variability of the cyclone paths towards the Mediterranean Sea. Inspection of the cyclone path in the simulation with orography and upper-level PV perturbation included showed that the cyclone ran into the orographic obstacle on the way to the Mediterranean Sea. The associated landrise (hill) the cyclone crossed was almost 500 meters, and was not experienced in other simulations of interest (f0, f1 and f3, refer to Eq. 3.2) to such an extent. Thus, this unique cyclone path, and the destruction over the hill could have been the reasons for the strong cyclolytic influence the analysed synergy tended to produce. This idea qualitatively resembles the studies on tropical cyclones passing over the island terrain (e.g. BENDER et al., 1987) that reported a strong cyclone filling as the cyclone passes the land disturbance. However, this cyclolytic influence lasted for 18 hours and it is not clear whether this type of orographically induced cyclone filling can be held responsible for the cyclolysis in the whole period.

There were significant differences noted in the times the cyclone reached the Mediterranean Sea. The two slowest cyclones were attached to orographically (f1, f12), while the two fastest ones to upper-level potential vorticity dominated simulations ( $\mathrm{f} 3, \mathrm{f} 23)$. As expected, sensible heat flux did not have a significant impact on the cyclone track variability. The aforementioned strong dependency indicates that the orographic influence was to keep the cyclone in the mountain lee, while upperlevel potential vorticity induced a faster advection of the low-level pressure system to the Mediterranean Sea.

\subsection{Sensitivity to thermal anomaly}

It was shown in the control run that the cyclone was attached to the small barotropic area in the beginning of the cyclogenesis. However, this area also corresponded to the strongest low-level PV values, associated with the strong primary PV banner near the SW edge of the mountain. In order to separate the influence of the thermal anomaly and low-level PV, a simulation with orography, but without thermal anomaly was performed.

In this simulation the thermal anomaly was removed from the initial conditions using the piecewise PVI technique (see section 4.2). Namely, it was shown (e.g. Hoskins et al., 1985) that a surface thermal anomaly can be regarded as an equivalent to a concentrated PV anomaly contained in a thin surface layer. The term surrogate $\mathrm{PV}$ has been introduced and widely used in referring to the surface potential temperature anomaly (e.g. REED et al., 1992; HuO et al., 1998), since the potential temperature anomaly is mathematically treated like a PV anomaly under the ground surface.

The difference between initial low-level temperatures of the sensitivity simulation and the control run is shown on Figure 9a. The broad Atlas-scale thermal anomaly perturbation reached $-4 \mathrm{~K}$ in the temperature field at $925 \mathrm{hPa}$, with associated mean sea level pressure increase of $5 \mathrm{hPa}$. Accordingly, the long baroclinic zone in the Atlas lee was significantly weakened. On a smaller scale, the shape of the isolines near the SW Atlas edge indicated weakening of the "secondary" thermal anomaly in the lee of the High Atlas, the place where the cyclone was initiated.

At 12 Nov 00 UTC, the approximate time of cyclogenesis commencement in the lee of the High Atlas, the initially removed thermal anomaly was strongly stretched out and already partly advected over the Mediterranean Sea with little effect in the Atlas lee (Fig. 9.b). This suggests that the atmospheric conditions in the lee of the High Atlas at cyclone initiation time were only slightly changed by the removal of the thermal anomaly perturbation. It can be verified from the figure that the relative differences in the area of interest reached up to $1 \mathrm{~K}$ and $1 \mathrm{hPa}$. The dipole structure of the thermal anomaly perturbation in the lee of the High Atlas denoted weakening of the baroclinic zone and frontally induced low-level PV before the cyclone commencement. Further inspection of the low-level atmospheric conditions at 12 Nov 00 UTC (Fig. 10a-b) showed that the surface baroclinic zone was somewhat weaker compared to the control run, in accordance with Figure 9.b, while the barotropic zone near the cyclone initiation point was more uniform. In this barotropic zone, a slightly weaker cyclone centre was located more inside the secondary thermal anomaly, closer to the mountain and orographic PV banner then in the control run. However, already at 12 Nov 12 UTC, there was no notable difference in the cyclone centre intensity or surface baroclinicity in the area of interest (Fig. 10c-d). Nevertheless, the cyclone centre was positioned closer to the mountain and continued to take that path in its further development (Fig. $9 b)$.

The simulation showed that a thermal anomaly in the lee of the Atlas built up rather quickly, practically during the 24-hour interval preceeding the cyclogenesis. Thus, little reliance could be made on the separation of lowlevel PV and thermal anomaly influences on the intensity of cyclone initiation in our simulation experiments. However, it seems that the location of the cyclone initiation showed sensitivity to thermal anomaly positioning in the mountain lee.

\section{Conclusions and final remarks}

This numerical sensitivity study investigated the initial phase of a deep Mediterranean cyclone that took place in the lee of the Atlas Mountains in November 2004 and caused a range of severe weather events throughout the Mediterranean region. In this numerical study, a factor 

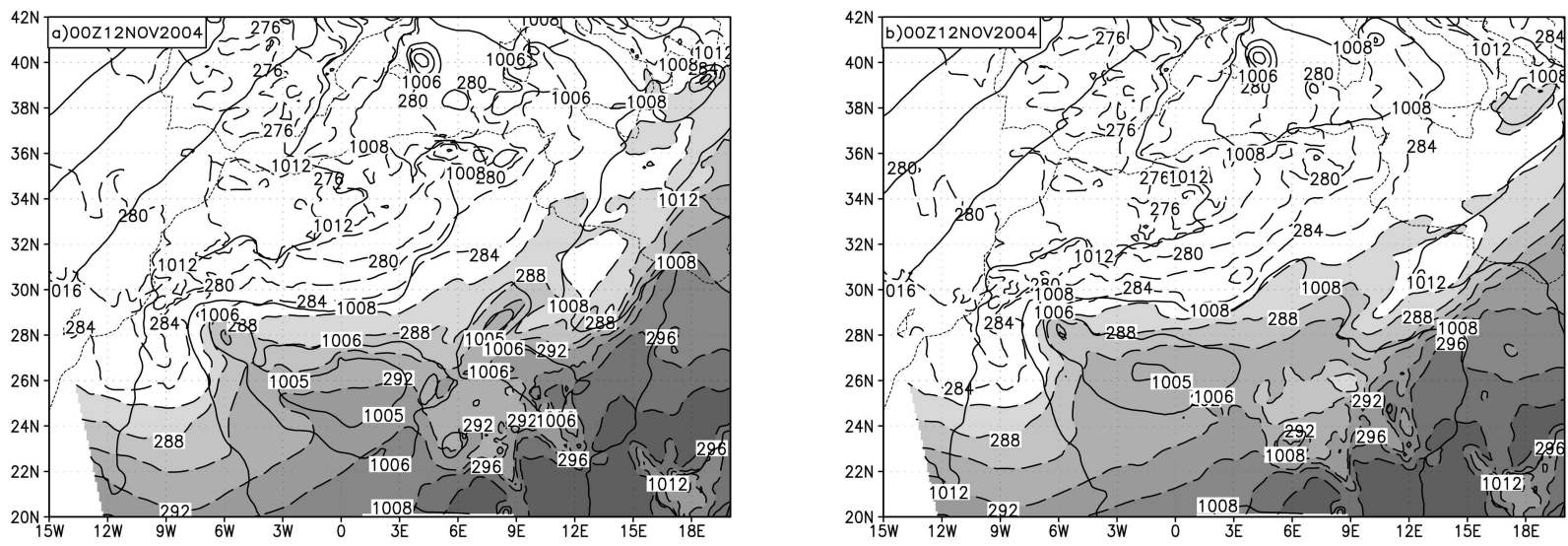

a)

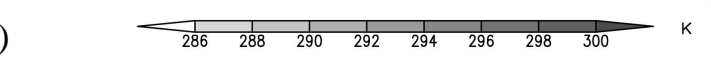

b)

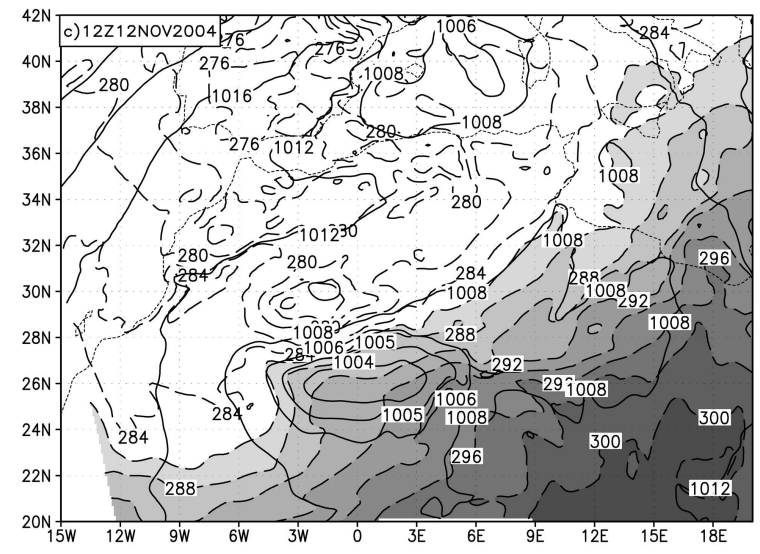

c)
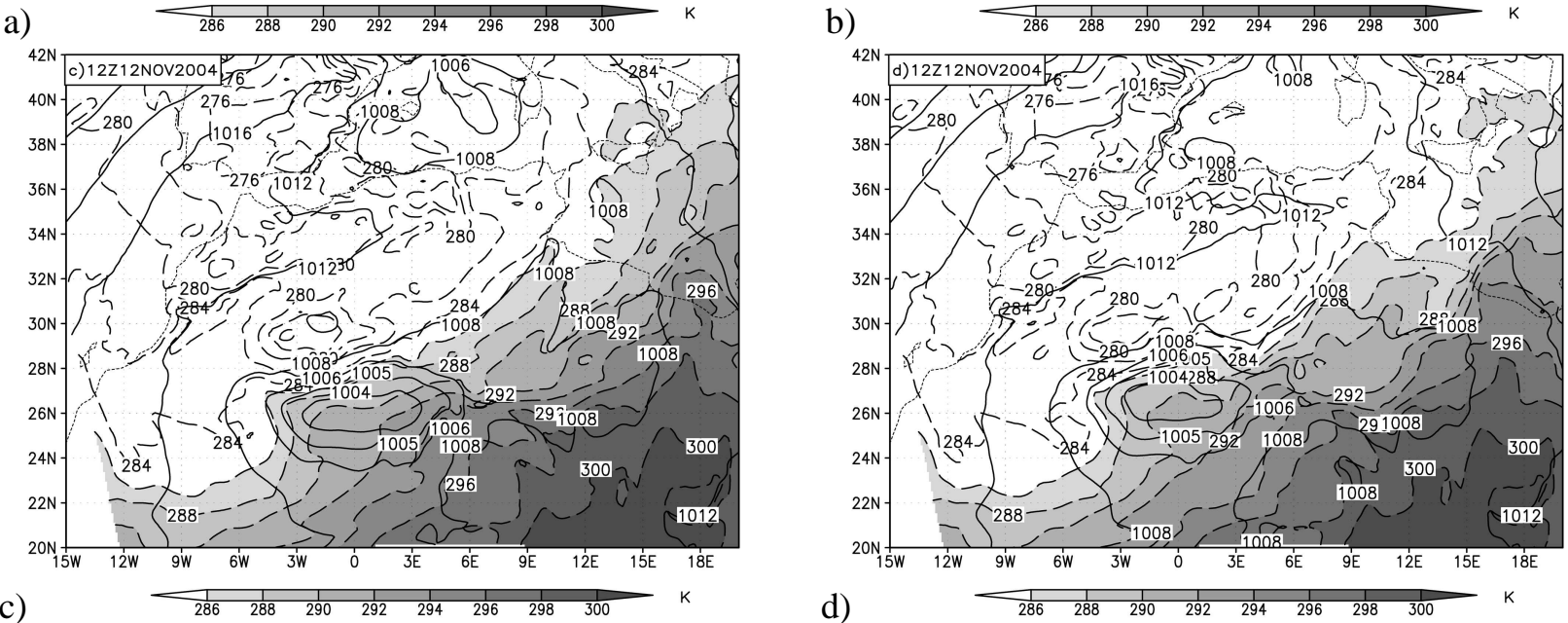

Figure 10: Model forecasts (shown on a zoomed domain) of the control run (a) and the thermal anomaly sensitivity run (b) at 12 Nov 2004 00 UTC and the control run (c) and the thermal anomaly sensitivity run (d) at 12 Nov 200412 UTC. Mean seal level pressure (hPa) is shown in continuous line (above $1008 \mathrm{hPa}$ every $4 \mathrm{hPa}$, under $1006 \mathrm{hPa}$ every $1 \mathrm{hPa}$ ). Temperature is plotted in dashed line (every $2 \mathrm{hPa}$ ), with shaded values over $286 \mathrm{~K}$.

separation method was applied to investigate the influences of orography, upper-level potential vorticity and surface sensible heat flux on the Atlas lee cyclone initiation and development, focused on the period until the cyclone left the lee area and entered the Mediterranean Sea.

Orography proved responsible for the first phase of the lee cyclogenesis, where a shallow cyclone was formed over a barotropic area in the lee of the High Atlas, due to frontal retardation and creation of an associated thermal anomaly. Although the orographic influence resembled the first stage of Alpine lee cyclogenesis, the orographically induced pressure drop reached 5 $\mathrm{hPa}$ during 24 hours and was not so rapid like in cases of Genoa cyclogenesis.

The second phase of the deepening was characterised by the cyclogenetic influence of the upper-level potential vorticity perturbation (a part of the upper-level PV anomaly). Indeed, with a total upper-level PV anomaly removed from the initial conditions, the cyclone did not form in the lee of the Atlas, that proves that the upper-level dynamical factors are a necessary in- gredient of Atlas lee cyclogenesis, in accordance with some idealised studies on Sharav lee cyclones (EGGER et al., 1995). In simulations with the PV perturbation included, the low-level air was colder, resulting in the creation of a stronger thermal anomaly and associated baroclinic zone. Furthermore, the upper-level PV perturbation showed responsible for a vertical (downward) propagation of cyclonic circulation, as indicated by the increased cyclonic winds in the lower atmosphere. In other words, the influence of positive PV advection contributed to the stronger low-level convergence and more rapid cyclone initiation.

It seems that the most pronounced feature of surface sensible heat flux contribution was an afternoon cyclolysis. Namely, SSHF was accompanied with increased vertical mixing in the afternoon ABL and associated weakening of the baroclinic (and barotropic) zone, inducing less intensive cyclone deepening. Secondly, heat exchange between the ground and the surface air reduced the horizontal surface air baroclinicity, as the horizontal air temperature gradient of the baroclinic zone was bigger then the horizontal ground temperature gradient in 
the arid Atlas lee. And finally, it is proposed that the exclusion of surface sensible heat flux enhanced the longwave earth radiation that tended to act as a heat supply to the moist areas (associated with the cyclone) in the free troposphere.

The synergy between orography and upper-level PV perturbation was at first cyclolytic, followed by the cyclogenetic contribution towards the end of the analysed period. The cyclolytic influence existed probably due to the fact that in the simulation with orography and upper-level PV included, the cyclone experienced filling over the terrain obstacle on the way to the Mediterranean Sea. The subsequent cyclogenetic contribution of the synergy had some potential for comparison with the low-level-upper-level vortex interaction in the second stage of Alpine lee cyclogenesis. However, at the end of the analysed period the cyclone centre positions differed significantly, not allowing a resolute conclusion.

The spread of the cyclone centres in the model simulations was shown to be a powerful tool in understanding the effect of different factors on the cyclone evolution. For instance, orography moved the cyclone initiation closer to the mountain and the favourable lee area where the thermal anomaly was the strongest and tended to keep the cyclone more stationary. On the other hand, the upper-level potential vorticity perturbation moved the cyclone formation location away from the mountain and was responsible for the faster advection of the cyclone to the Mediterranean Sea. It seems that the intensity of the upper-level PV anomaly has an influence on the subsequent cyclone movement and possibly has some potential to account for the seasonal cyclone path variability.

Another sensitivity experiment was performed to try to account for the influence of a thermal anomaly on the generation of a low-level vortex in the first stage of lee cyclogenesis. However, the simulation showed that the thermal anomaly in the lee of the Atlas built up rather quickly. It almost reached the intensity of the thermal anomaly of the control run in 24 hours since the start of the simulation, just before the cyclogenesis started. Thus, little reliance could be made on the separation of low-level PV and thermal anomaly influences on the intensity of cyclone initiation. However, it seems that the location of the slightly weaker cyclone initiation showed sensitivity to a thermal anomaly positioning in the mountain lee.

So far, the very area of the Atlas lee was subjected neither to a significant number of numerical or theoretical studies, nor to observational field campaigns like MAP. However, the arid lee area of the Atlas Mountains has some potential to attribute more precisely the influence of dynamical processes to the lee cyclogenesis due to the lack of moist processes at play. Furthermore, the vertical and horizontal scales of the High Atlas Moun- tains are reasonably comparable to the Alpine dimensions.

It is one of the goals of the planned MEDEX special field campaign to better monitor Mediterranean cyclones and storms, which are often not well detected by observation networks. In addition, this effort should be accompanied by an increased number of numerical and theoretical studies, specially the ones that investigate the life cycles of the high-impact deep Mediterranean cyclones.

\section{Acknowledgements}

The work of Kristian HORVATH has been supported by the Ministry of Science, Education and Sports of the Republic of Croatia under the project number 0004001 and Ministerio de Medio Ambiente of Spain (Instituto Nacional de Meteorologia) scholarship grant. The work of Lluis FITA has been supported by the MEDEXIB: REN 2002-03482 grant. Authors are grateful to the anonymous referees for their valuable comments and suggestions, that improved the content of this paper. Valuable help in the analysis of the satellite pictures and preparation of the manuscript was received from Natasa Strelec-Mahovic, Luksa Kraljević and Ivana STIPERSKI from Meteorological and Hydrological Service of Croatia.

\section{References}

AEBISCHER, U., C. SCHÄR, 1998: Low-Level Potential Vorticity and Cyclogenesis to the Lee of the Alps. - J. Atmos. Sci. 55, 186-207.

AlPERT, P., B. ZIV, 1989:The Sharav cyclone - Observations and some theorethical considerations. - J. Geophy. Res. 94, 18495-18514.

AlPERT, P., B. NeEmAn, Y. ShaY-EL, 1990: Intermonthly variability of cyclone tracks in the Mediterranean. - J. Climate, 3, 1474-1478.

Alpert, P., U. Stein, M. Tsidulko, 1995: Role of sea fluxes and topography in Eastern Mediterranean cyclogenesis. - The Global Atmosphere and Ocean System 3, 55-79.

Alpert, P., M. Tsidulko, S. KrichaK, U. Stein, 1996: A multi-stage evolution of an ALPEX cyclone. - Tellus 48A, 209-220.

Bender, M.A., R.E. Tuleya, Y. Kurihara, 1987: A numerical study of the effect of island terrain on tropical cyclones. - Mon. Wea. Rev. 115, 130-155.

BERGER, A., 2001: The role of $\mathrm{CO}_{2}$, sea level and vegetation during Milankovitch-forced glacial-interglacial cycles. - In: Geosphere-biosphere interactions and climate. Bengtsson, C.U. HAMmeR (Eds). Cambridge University Press, 119-146.

Betts, A. K., M. J. Miller, 1993: The Betts-Miller scheme. - In: The representation of cumulus convection in numerical models. K. A. EMANUEL, D. J. RAYMOND (Eds). Amer. Meteor. Soc. 107-121. 
Blackadar, A. K., 1979: High resolution models of the planetary boundary layer. - Advances in Environmental Science and Engineering 1, No. 1. J. PfAfFlin, E. Ziegler (Eds). Gordon and Breach, 50-85.

Bretherton, F. P., 1966: Baroclinic instability and the short wavelength cut-off in terms of potential vorticity. - Quart. J. Roy. Meteor. Soc. 92, 335-345.

Buzzi, A., S. Tibaldi, 1978: Cyclogenesis in the lee of Alps: A case study. - Quart. J. Roy. Meteor. Soc. 104, 271287.

Charney, J. G., 1955: The use of primitive equations of motion in numerical prediction. - Tellus 7, 22-26.

Conte, M., 1985: The meteorological "bomb" in the Mediterranean: a synoptic climatology. - Report of the Steering group meeting on the Mediterranean Cyclones Study Project, PSPM Report Series No. 20, WMO/TD No. 128, 17-31.

Davis, C. A., K. Emanuel, 1991: Potential vorticity diagnostics of cyclogenesis. - Mon. Wea. Rev. 119, 1929-1953.

Dell'osso, L., DJ. RAdinovic, 1984: A case study of cyclone development in the lee of Alps on 18 March 1982. Contrib. Atmos. Phys. 57, 369-379.

DudhiA, J., 1993: A nonhydrostatic version of the Penn State/NCAR mesoscale model: Validation tests and simulation of an Atlantic cyclone and cold front. - Mon. Wea. Rev. 121, 1493-1513.

—, 1996: A multi-layer soil temperature model for MM5. Preprints. - The $6^{\text {th }}$ PSU/NCAR Mesoscale Model Users' Workshop, 22-24 July 1996, Boulder, Colorado, 49-50.

Egger, J., P. Alpert, A. TAfferner, B. Ziv, 1995: Numerical experiments on the genesis of Sharav cyclones: Idealized simulations. - Tellus 47A, 162-174.

Grell, G. A., J. DudhiA, D.R. STAuffer, 1994: A description of the fifth-generation Penn State/NCAR mesoscale model (MM5). - NCAR Techincal Note, NCAR/TN-398+STR, 117 pp.

GUAN, S., G. W. REUTER, 1996: Numerical simulation of an industrial cumulus affected by heat, moisture and CCN released from oil refinery. - J. Appl. Meteor. 35, 1257-1264.

Hoskins, B. J., M. E. Mcintyre, A. W. Robertson, 1985: On the use and significance of isentropic potential vorticity maps. - Quart. J. Roy. Meteor. Soc. 111, 877-946.

HuO, Z., D.-L. ZhAng, J. GyAKUM, 1998: An Application of Potential Vorticity Inversion to Improving the Numerical Prediction of the March 1993 Superstorm. - Mon. Wea. Rev. 126, 424-436.

KrichaK, S. O., P. Alpert, T. N. Krishnamurti, 1997a: Interaction of topography and tropospheric flow - A possible generator for the Red Sea trough? - Meteor. Atmos. Phys. 63, 149-158.

KrichaK, S. O., P. Alpert, T. N. KRishnamurti, 1997b: Red Sea trough/cyclone development numerical investigation. - Meteor. Atmos. Phys. 63, 159-170.

KUO, Y.-H., R. J. REED, S. LOW-NAM, 1991: Effects of surface energy fluxes during the early development and rapid intensification stages of seven explosive cyclones in the Western Atlantic. - Mon. Wea. Rev. 119, 457-475.

KuO, Y.-H., J. R. GyaKuM, Z. GuO, 1995: A case of rapid continental mesoscale cyclogenesis. Part I: Model sensitivity experiments. - Mon. Wea. Rev. 123, 970-997.

Lagouvardos, K., V. Kotroni, 2000: Use of the METEOSAT water vapour images for the diagnosis of a vig- orous stratospheric intrusion over central Mediterranean. Meteor. Appl. 7, 205-210.

MANSFIELD, D., 2001: Project III.1: IPV analysis in relation to satellite images. - In: LAGOUVARDos, K., E. LiLJAS, B. Conway, J. Sunde, (Eds): Improvement of nowcasting techniques, COST Action $\mathbf{7 8}$ Final Report. European Commission, 189-201.

MCGinley, J., 1982: A Diagnosis of Alpine Lee Cyclogenesis. - Mon. Wea. Rev. 110, 1271-1287.

PedGley, D., 1972: Desert depression over north-east Africa. - Met. Mag. 101, 228-243.

Pettersen, S., S. J. Smebye, 1979: On the development of extratropical cyclones. - Quart. J. Roy. Meteor. Soc. 97, $457-482$.

Phillips, N. A., 1954: Energy transformations and meridional circulations associated with simple baroclinic waves in a two-level, quasi-geostrophic model. - Tellus 6, 273286.

Prezerakos, N. G., 1985: The north-west African depressions affecting the southern Balkans. - J. Climatol. 5, 643654.

—, 1990: Synoptic flow patterns leading to the generation of north-west African depressions. - Int. J. Climatol. 10, 3347.

Prezerakos, N. G., S. C. Michaelides, A. S. Vlassi, 1990: Atmospheric synoptic conditions associated with the initiation of north-west African depressions. - Int. J. Climatol. 10, 711-729.

RADINOVIC, D.J., 1987: Mediterranean cyclones and their influence on the weather and climate. - WMO PSMP Rep. 24, 131 pp.

Reed, R. J., M. T. StoelingA, Y.-H. Kuo, 1992: A Model-aided Study of the Origin and Evolution of the Anomalously High Potential Vorticity in the Inner Region of a Rapidly Deepening Marine Cyclone. - Mon. Wea. Rev. 120, 893-913.

ROMERO, R., 2001: Sensitivity of a heavy rain producing Western Mediterranean cyclone to embedded potential vorticity anomalies. - Quart. J. Roy. Meteor. Soc. 127, 25592597.

Romero, R., C. RAMis, S. Alonso, 1997: Numerical simulation of an extreme rainfall event in Catalonia: Role of orography and evaporation from the sea. - Quart. J. Roy. Meteor. Soc. 123, 537-559.

Stein, U., P. AlPERT, 1993: Factor separation in numerical simulations. - J. Atmos. Sci. 50, 2107-2115.

Thorncroft, C. D., H. A. Flocas, 1997: A Case Study of Saharan Cyclogenesis. - Mon. Wea. Rev. 125, 1147-1165.

TSIDUlKo, M., P. AlPERT, 2001: Synergism of upper-level potential vorticity and mountains in Genoa lee cyclogenesis - A numerical study. - Meteor. Atmos. Phys. 78, 261-285.

ZhANG, D. L., R. A. Anthes, 1982: A high-resolution model of the planetary boundary layer. - Sensitivity tests and comparison with SESAME-79 data. - J. Appl. Meteor. 21, 1594-1609.

ZHANG, D. L., J. M. FRITSCH, 1986: Numerical simulation of the meso- $\beta$ scale structure and evolution of the 1977 Johnstown flood. Part I: Model description and verification. - J. Atmos. Sci. 43, 1913-1943. 